\title{
A CORNEO-SCLERAL SUTURE IN CATARACT EXTRACTION. ITS TECHNIQUE AND ADVANTAGES
}

\author{
BY \\ H. B. STALlaRd \\ LONDON
}

THE use of a corneo-scleral suture in the operation for cataract extraction has been advocated in the past by a number of ophthalmic surgeons in different countries, and there are many modifications in the manner in which this suture is applied. The literature on this matter does not comment fully on the advantages to be gained by this addition to the technique of cataract surgery.

To date I have never seen it noted that a corneo-scleral suture properly applied is of considerable value in the prevention of postoperative hyphaema. In most cases this complication occurs about the 4th or 5th day after operation and the blood comes from the edge of the section wound, probably from opening of the wound and the tearing of the newly formed capillaries which traverse it.

In 107 consecutive cases of cataract extraction (79 extra-capsular and 28 intra-capsular) in which I have used a corneo-scleral suture in the manner described below there has been no post-operative hyphaema, whereas before using this stitch this complication was present in about 30-35 per cent. of the cataract extractions I did in the Moorfields Eye Hospital. This disparity is too remarkable to be explained on fortuitous grounds.

The corneo-scleral suture is particularly of value in elderly persons for whom immobilisation in bed for a few days may have adverse consequences. The patient may with safety be allowed out of bed into a chair on the day following operation. The stitch is probably a factor in the prevention of iris prolapse. In 2 of the series of 107 cases a narrow rim of iris was seen in the wound24-48 hours after operation, in one case on the nasal side and in the other on the temporal. In both these cases I was conscious of the fact that I had not tied the corneo-scleral stitch with that degree of firmness which is proper, and in one the iris was flaccid and difficult to replace so that it was tempting providence not to have done an iridectomy.

In the extra-capsular operation I have generally left the eye without a peripheral iridectomy but have preferred to do this as a preliminary to intra-capsular extraction. Graves has pointed out the use that may be made of the scleral part of the suture for the purpose of fixation of the eye. This may be effected by holding 
together in plane forceps the two ends of the scleral part of the suture 1 or $2 \mathrm{~mm}$. from their point of passage through the sclera. This mode of fixation of the globe is particularly good in elderly patients whose conjunctiva is thin and atrophic and would tear quickly in the fixation forceps.

The corneo-scleral suture is of considerable value in cases where vitreous loss may be anticipated, on account of a degenerated state of this media, a highly myopic eye, and intra-capsular extraction. I think that it is the only surgical manoeuvre that will in any way check that relentless forward flow which occurs once the vitreous face has been broken. Stitches in the conjunctival flap are useless when this disaster occurs. No untoward consequences have occurred in the series of 107 cases in which this stitch has been used. Some transient striate keratitis in the vicinity of the stitch has been present in some cases, but this has never been so severe as in some cases of cataract extraction in which the stitch has not been used. It clears up in 8-10 days and leaves no permanent defect. There has been no case of corneal abscess or ulceration arising from the use of this suture.

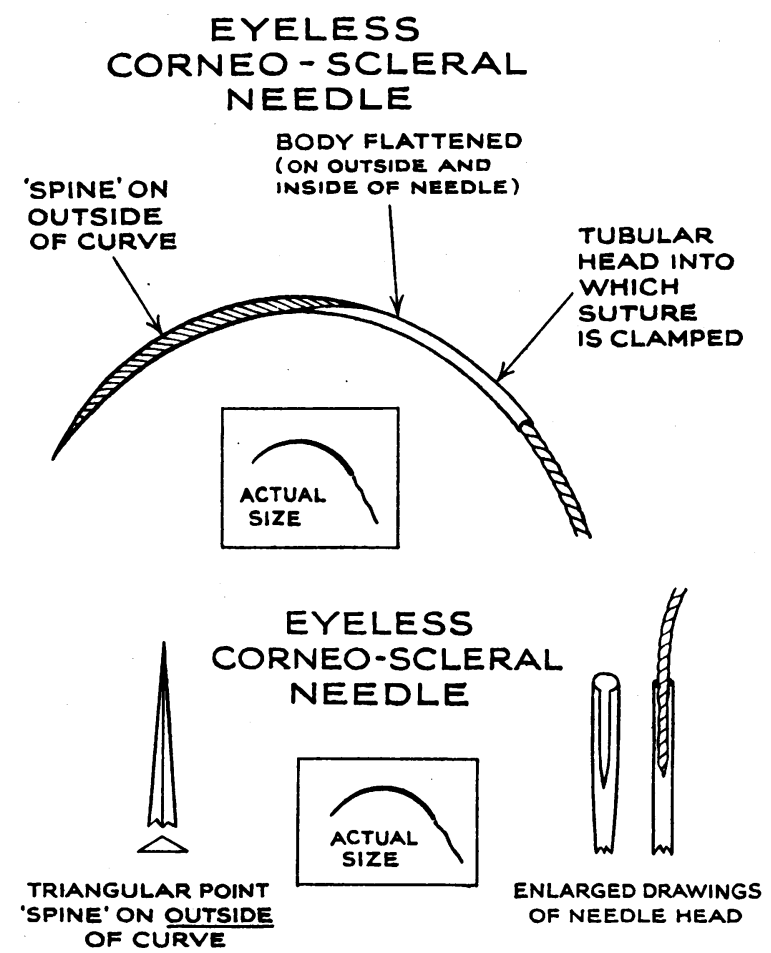

FIG. 1. 
In most cases the astigmatism has varied between 1.25 and 2.25 D., in a very few cases it was 3 or 325 D., and in some there was no post-operative astigmatism at all.

Technique.-The needle and suture (2/0 black braided silk) were made for me by Merson Ltd., Edinburgh. The needle is $12 \mathrm{~mm}$. long, the curve corresponds approximately to that of the Maddox needle but unlike this needle it is eyeless and the spine has a cutting edge on the convex or outer aspect of the needle (the spine of the Maddox needle is on its concave inner aspect) (see Fig. 1). It is held in a small Quarry Silcock's needle holder, the jaws of which are fined down.

Preliminary to insertion of the suture, akinesia of the orbicularis is produced, a stitch is passed through the skin of the upper lid $3 \mathrm{~mm}$. from the centre of the lid margin, an Arruga's speculum (see Fig. 2) is inserted and a superior rectus stitch is hitched and

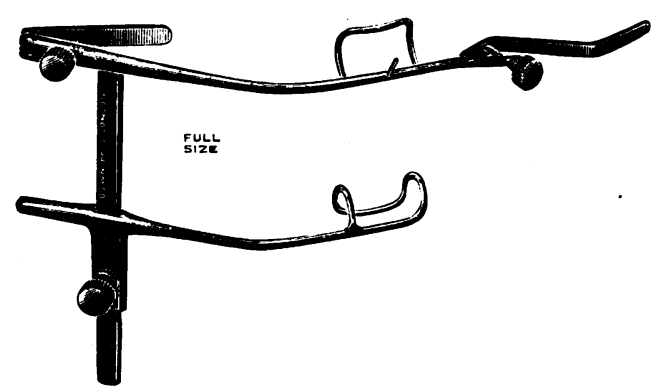

Fig. 2.

Arruga's Speculum (Down Bros., London).

tied over a small boss on the upper shank of the speculum. At this stage the upper shank of the speculum should not be racked up by the screws controlling the thin plates that rest on the bridge of the nose and the temporal region. If the upper shank of the speculum is thus lifted away from the globe a difficult angle is made for manipulating the needle holder when passing the corneo-scleral suture.

The globe is partly steadied and fixed by the superior rectus stitch but additional fixation is needed by forceps applied so as to grasp the insertion of the internal rectus and overlying conjunctiva when the stitch is passed through the right cornea and the external rectus in the case of the left eye, the fixation forceps being held in the left hand and the needle holder in the right when passing the suture in each eye.

When the eye is fixed the point of the needle is dipped into the anterior half of the substantia propria of the cornea at a point 
$1 \mathrm{~mm}$. inside the limbus in the " 12 o'clock" meridian. The needle is made to traverse 2 to $2.5 \mathrm{~mm}$. of the cornea in a transverse direction before emerging through the surface. The surgeon still maintains fixation of the globe whilst the needle is inserted in the holder with its point facing in the opposite direction to that in which it traversed the cornea.

The point of the needle is then passed through the conjunctival and subconjunctival tissues and engages in the superficial layers of the sclera $1.5 \mathrm{~mm}$. above the limbus opposite the site at which the needle emerged from the cornea. It passes transversely through the superficial half of the sclera for $25 \mathrm{~mm}$. and is brought out

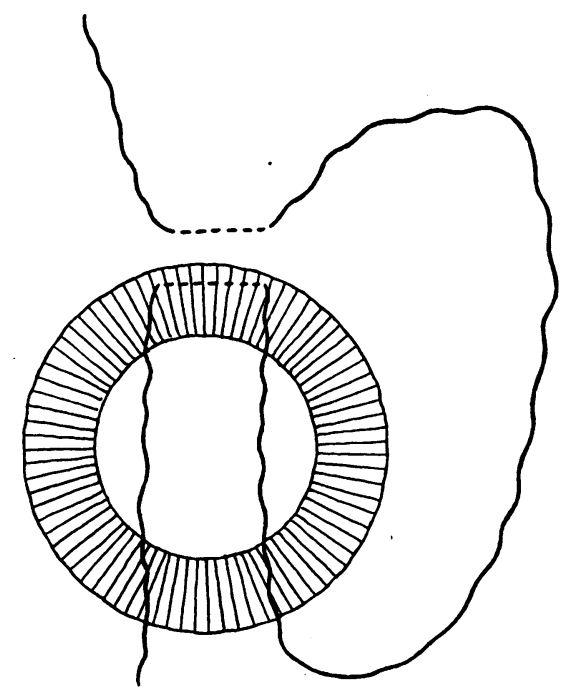

FIG. 3.

through the conjunctiva opposite its entry wound in the cornea. The suture is damped with sterile saline and the corneal ends of the stitch are then drawn downwards in the " 6 o'clock" meridian are placed over the cornea and the scleral ends are drawn upwards in the " 12 o'clock" meridian and laid on the conjunctiva (see Fig. 3).

The screws on Arruga's speculum are then adjusted so that the upper shank is held away from the eye and the weight of the speculum is borne on the bridge of the nose and the temporal region.

The corneo-scleral section is made and the Graefe knife cuts out between the corneal and scleral parts of the suture. It is generally possible to obtain a small frill of conjunctiva as a flap. The corneal ends of the suture are held in iris forceps and gently 
drawn forwards so as to open the wound sufficiently to admit a pair of capsule forceps.

In the intra-capsular operation immediately the lens is delivered the scleral end of the suture is drawn through so that the edges of the section wound are closely and firmly coapted. The suture is then tied with two forceps. In the extra-capsular operation the scleral end of the suture should be drawn through, but 2-3 $\mathrm{mm}$. of the loop should be allowed to remain slack so that the edges of the section wound may be separated enough to allow the introduction of a cannula for washing out the soft lens matter from the anterior chamber.

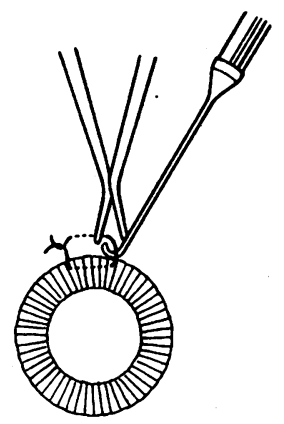

FIG. 4.

Care must be taken to see that the conjunctival frill is not turned into the section wound when the stitch is tied. The frill of conjunctiva is stroked into place with an iris repositor.

The stitch is removed on the 14th day after operation. Pantocain 1 per cent. is instilled and the upper lid held away from the globe with a Desmarres' retractor. The loop opposite the knot (see Fig. 4) is seized with straight plane iris forceps or lifted up very gently on a small hook and divided by de Wecker's scissors which have been ground down and have blunt ends. The knot is then seized with iris forceps and the suture withdrawn carefully and gently in the plane of its transverse course.

Summary.-The advantages of a corneo-scleral suture in the operation of cataract extraction, both intra-capsular and extracapsular, are discussed. This suture is particularly of value in the prevention of post-operative hyphaema. In a series of 107 cases (79 extra-capsular and 28 intra-capsular) this complication did not occur at all.

The needle employed and the technique of inserting the suture are described. 